\title{
Site testing for submillimetre astronomy at Dome C, Antarctica
}

\author{
P. Tremblin ${ }^{1}$, V. Minier ${ }^{1}$, N. Schneider ${ }^{1}$, G. Al. Durand ${ }^{1}$, M. C. B. Ashley ${ }^{2}$, J. S. Lawrence ${ }^{2}$, D. M. Luong-Van ${ }^{2}$, \\ J. W. V. Storey ${ }^{2}$, G. An. Durand ${ }^{3}$, Y. Reinert ${ }^{3}$, C. Veyssiere ${ }^{3}$, C. Walter ${ }^{3}$, P. Ade ${ }^{4}$, P. G. Calisse ${ }^{4}$, Z. Challita ${ }^{5,6}$, \\ E. Fossat ${ }^{6}$, L. Sabbatini ${ }^{5,7}$, A. Pellegrini ${ }^{8}$, P. Ricaud ${ }^{9}$, and J. Urban ${ }^{10}$ \\ ${ }^{1}$ Laboratoire AIM Paris-Saclay (CEA/Irfu, Univ. Paris Diderot, CNRS/INSU), Centre d'études de Saclay, 91191 Gif-Sur-Yvette, \\ France \\ e-mail: [pascal.tremblin; vincent.minier]@cea.fr \\ 2 University of New South Wales, 2052 Sydney, Australia \\ 3 Service d'ingénierie des systèmes, CEA/Irfu, Centre d'études de Saclay, 91191 Gif-Sur-Yvette, France \\ 4 School of Physics \& Astronomy, Cardiff University, 5 The Parade, Cardiff, CF24 3AA, UK \\ 5 Concordia Station, Dome C, Antarctica \\ ${ }^{6}$ Laboratoire Fizeau (Obs. Côte d'Azur, Univ. Nice Sophia Antipolis, CNRS/INSU), Parc Valrose, 06108 Nice, France \\ 7 Departement of Physics, University of Roma Tre, Italy \\ 8 Programma Nazionale Ricerche in Antartide, ENEA, Rome Italy \\ 9 Laboratoire d'Aérologie, UMR 5560 CNRS, Université Paul-Sabatier, 31400 Toulouse, France \\ ${ }^{10}$ Chalmers University of Technology, Department of Earth and Space Sciences, 41296 Göteborg, Sweden
}

Received 25 May 2011 / Accepted 17 October 2011

\begin{abstract}
Aims. Over the past few years a major effort has been put into the exploration of potential sites for the deployment of submillimetre astronomical facilities. Amongst the most important sites are Dome C and Dome A on the Antarctic Plateau, and the Chajnantor area in Chile. In this context, we report on measurements of the sky opacity at $200 \mu \mathrm{m}$ over a period of three years at the French-Italian station, Concordia, at Dome C, Antarctica. We also present some solutions to the challenges of operating in the harsh polar environment. Methods. The 200- $\mu \mathrm{m}$ atmospheric opacity was measured with a tipper. The forward atmospheric model MOLIERE (Microwave Observation LIne Estimation and REtrieval) was used to calculate the atmospheric transmission and to evaluate the precipitable water vapour content (PWV) from the observed sky opacity. These results have been compared with satellite measurements from the Infrared Atmospheric Sounding Interferometer (IASI) on Metop-A, with balloon humidity sondes and with results obtained by a ground-based microwave radiometer (HAMSTRAD). In addition, a series of experiments has been designed to study frost formation on surfaces, and the temporal and spatial evolution of thermal gradients in the low atmosphere.

Results. Dome $\mathrm{C}$ offers exceptional conditions in terms of absolute atmospheric transmission and stability for submillimetre astronomy. Over the austral winter the PWV exhibits long periods during which it is stable and at a very low level (0.1 to $0.3 \mathrm{~mm})$. Higher values $(0.2$ to $0.8 \mathrm{~mm})$ of PWV are observed during the short summer period. Based on observations over three years, a transmission of around $50 \%$ at $350 \mu \mathrm{m}$ is achieved for $75 \%$ of the time. The $200-\mu \mathrm{m}$ window opens with a typical transmission of $10 \%$ to $15 \%$ for $25 \%$ of the time.

Conclusions. Dome $\mathrm{C}$ is one of the best accessible sites on Earth for submillimetre astronomy. Observations at 350 or $450 \mu \mathrm{m}$ are possible all year round, and the 200- $\mu \mathrm{m}$ window opens long enough and with a sufficient transparency to be useful. Although the polar environment severely constrains hardware design, a permanent observatory with appropriate technical capabilities is feasible. Because of the very good astronomical conditions, high angular resolution and time series (multi-year) observations at Dome $\mathrm{C}$ with a medium size single dish telescope would enable unique studies to be conducted, some of which are not otherwise feasible even from space.
\end{abstract}

Key words. site testing - atmospheric effects - submillimeter: general

\section{Introduction}

Submillimetre (submm) astronomy is one of the most important techniques for the study of the cold Universe and for unveiling the birth and early evolution of planets, stars, and galaxies. Submm continuum observations are particularly powerful for the measurement of luminosities, temperatures, and masses of cool, dusty objects because dust enshrouded star-forming regions emit the bulk of their energy between 60 and $500 \mu \mathrm{m}$. The submm range of the spectrum (or $\mathrm{THz}$ regime in frequency) is also rich in the atomic and molecular lines that offer the only means of studying the kinematic structure of the interstellar medium of galaxies. Observations at these wavelengths with medium to large telescopes should lead to breakthroughs in the study of star-formation at all scales, and to a better understanding of its history back to the early Universe-thus leading to a better understanding of galaxy evolution. Asteroids, debris disks, planet formation, dust origin in evolved stars, interstellar dust and polarisation of dust in the Universe are also potential science drivers for submm astronomy (see Minier et al. 2009, for details).

\subsection{Dome $C$, a potential site}

A major obstacle to ground-based observations in the submm range (and specifically at wavelengths shorter than $500 \mu \mathrm{m}$ ) is 
the atmosphere. This part of the electromagnetic spectrum is normally the preserve of space telescopes such as the Herschel space observatory (Pilbratt et al. 2010) although large submm facilities such as ALMA will be able to operate down to $420 \mu \mathrm{m}$ and possibly below in the future (Hills et al. 2010). However, submm observations in the $200-\mu \mathrm{m}$ window with ground-based instruments will always require exceptional conditions (see Marrone et al. 2005; Oberst et al. 2006). A very good, highaltitude site like Chajnantor is usable for less than $25 \%$ of the time in winter (i.e. at least $40 \%$ transmission) in the $350 / 450 \mu \mathrm{m}$ windows, and probably for less than $5 \%$ of the time at $200 \mu \mathrm{m}$ (Peterson et al. 2003). Matsushita et al. (1999) reported a more optimistic estimate, operational experience with APEX, ALMA, and other telescopes will tell.

While ground-based observations are limited by the atmosphere, space telescopes remain limited in size and thus can offer only modest angular resolution: from $\sim 6^{\prime \prime}$ to $37^{\prime \prime}$ at 70 to $500 \mu \mathrm{m}$, respectively for the Herschel space observatory, implying a fairly high extragalactic confusion limit and preventing the study of individual protostars in all but the nearest starforming clusters of our Galaxy (e.g. Schneider et al. 2010; André et al. 2010). In this context, large (>10 m) single-dish telescopes operating at $200-450 \mu \mathrm{m}$, can provide a better angular resolution than Herschel, and wider-field mapping capabilities than ALMA. This appears to satisfy a clear need in submm astronomy (e.g. Radford et al. 2008). As a result, an intensive study of new sites is currently underway.

Although the average altitude of the Antarctic plateau, $\sim 3000 \mathrm{~m}$, is less than that of the Chajnantor plateau $(\sim 5000 \mathrm{~m})$, Antarctica might nevertheless offer better conditions for submm astronomy because of its peculiar geography and climate. The very low atmospheric precipitable water vapour content (PWV) in Antarctic results from the low sun elevation, isolation by the circumpolar vortex, and the high optical reflectivity of ice combined with its high emissivity in the infrared-leading to intense radiative cooling of the ice at night. These phenomena make Antarctica the coldest continent on Earth. Snow precipitation is very low on the Antarctic plateau and low pressure fronts rarely penetrate into the inner plateau, instead staying at the coastline. In fact, the inland part of the continent is a true desert: across an area of 5 million $\mathrm{km}^{2}$, snow precipitation is about $5 \mathrm{~cm}$ annually, and often less than $2 \mathrm{~cm}$ at the highest regions (called Domes). As a consequence, the average PWV above the Antarctic plateau is expected to be lower than at Chajnantor (Minier et al. 2009, and references therein).

These high plateau Antarctic sites (Domes A, C, F) are therefore potential locations for astronomical facilities (for Dome A see Yang et al. 2010). The relative merits of the sites have been presented in Saunders et al. (2009). These studies follow the development of astronomy at the South Pole, where submm facilities have already been deployed by US astronomers. The atmospheric transmission at $225 \mathrm{GHz}$ has been measured there in 1992 with an NRAO radiometer (Chamberlin 2004). The pathfinder for Antarctic submm-astronomy was AST/RO, a $1.7 \mathrm{~m}$ dish (Stark et al. 2001) installed at the South Pole and operational from 1995. Recently, a $10 \mathrm{~m}$ submm dish has been built at the South Pole (South Pole Telescope SPT, Carlstrom et al. 2011).

Dome $\mathrm{C}$ is the location of the French-Italian Concordia station, accessible from both the Dumont-d'Urville and Mario Zucchelli coastal stations by either light plane or, for transporting heavy material, motorised ground expeditions. Concordia station hosts a crew of people during winter and thus allows experiments all year round. Monitoring of the atmosphere

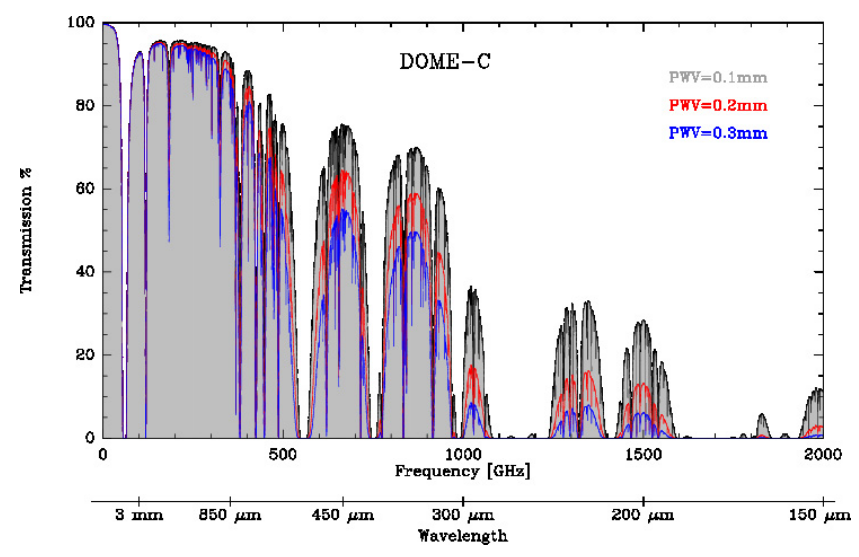

Fig. 1. Atmospheric transmission at $0.1,0.2$, and $0.3 \mathrm{~mm}$ PWV between $0-2 \mathrm{THz}(\approx 3 \mathrm{~mm}$ to $\approx 150 \mu \mathrm{m})$ at Dome $\mathrm{C}$ calculated with the atmospheric model MOLIERE.

characteristics and qualification of the site for optical and nearinfrared astronomy have been conducted for many years by French, Italian, and Australian teams (e.g. Aristidi et al. 2005; Lawrence et al. 2004). However, little effort has so far been put into evaluation of the quality of the atmosphere, the meteorological constraints, or the specific advantages of Dome $\mathrm{C}$ for a potential submm/FIR telescope. Preliminary meteorological studies and atmospheric transmission models (Schneider et al. (2009) and Fig. 1) suggest that Dome $\mathrm{C}$ might offer atmospheric conditions that open the 200- $\mu$ m window (Valenziano \& Dall'Oglio 1999; Tomasi et al. 2006; Minier et al. 2008) for a significant amount of time. Calisse et al. (2004) undertook measurements of the atmospheric opacity at $350 \mu \mathrm{m}$ during a summer campaign and found that it is comparable, but consistently better than, that at South Pole. However, no direct assessment of the wintertime atmospheric transmission has so far been performed.

The stability of the atmosphere is an equally important parameter when comparing different sites, and Dome $\mathrm{C}$ may turn out to be far more stable than the Chilean sites (Minier et al. 2008). Determining whether a telescope facility at Dome $C$ in Antarctica might be able to operate in all atmospheric windows between $200 \mu \mathrm{m}$ and $1 \mathrm{~mm}$, and routinely at 350 and $450 \mu \mathrm{m}$ throughout the year, has been the main objective of our site testing activities since 2008, as reported in the ARENA $^{1}$ European conference proceedings.

\subsection{Prerequisites for a large submm telescope at Dome $C$}

A study of the necessary pre-requirements for the future deployment of a large, submm telescope has been carried out between 2007 and 2010 at Concordia. Besides the atmospheric transmission in all windows in the far-infrared and submillimetre parts of the spectrum, complementary site testing has focused on the polar constraints and their potential impact on instruments. This work includes a study of the removal and prevention of frost formation, and a study of the temperature gradient in the ground layer.

\section{Ground-based measurement of atmospheric transmission}

The sky opacity at submillimetre wavelengths depends critically on the amount of water vapour in the atmosphere. SUMMIT08

\footnotetext{
1 Antarctic Research, a European Network for Astrophysics: http: //arena.unice. fr
} 
(SUbMilliMetre Tipper version 2008) is an in situ instrument measuring sky transparency at $200 \mu \mathrm{m}$. It consists of a motorised mirror collecting atmospheric radiation at different elevations. This flux is then measured by a photometer behind a $200-\mu \mathrm{m}$ filter. The previous prototype, SUMMIT, was installed in 2000 at Concordia by the University of New South Wales (UNSW) and performed measurements during the Austral summer between December 2000 and January 2001 (Calisse et al. 2004). SUMMIT has then been refurbished in Saclay and was installed at Concordia in February 2008 under the responsability of CEA/Irfu in collaboration with UNSW (Durand et al. 2008). The instrument then worked almost continuously between April 2008 and December 2008, and has been in full operation since June 2009.

\subsection{The atmospheric model MOLIERE}

MOLIERE (Microwave Observation and LIne Estimation and REtrieval) is a forward and inversion atmospheric model (Urban et al. 2004), developed for atmospheric science applications. The code calculates the absorption of radiation in the $\mathrm{mm}$ - to mid-IR wavelength range (equivalent from 0 to $10 \mathrm{THz}$ in frequency) as a result of several effects: (i) spectroscopic lines such as those of atmospheric water $\left(\mathrm{H}_{2} \mathrm{O}\right)$, oxygen $\left(\mathrm{O}_{2}\right)$, and ozone $\left(\mathrm{O}_{3}\right)$ absorb strongly at short wavelengths, while (ii) collisions of $\mathrm{H}_{2} \mathrm{O}$ with $\mathrm{O}_{2}$ and nitrogen $\left(\mathrm{N}_{2}\right)$ result in continuous absorption across all wavelengths. For the line absorption, a radiative transfer model including refraction and absorption by major and minor atmospheric species is included $\left(\mathrm{H}_{2} \mathrm{O}, \mathrm{O}_{2}, \mathrm{O}_{3}, \mathrm{NO}_{2}, \mathrm{HNO}_{3}, \mathrm{CO}\right.$ and other lines up to $10 \mathrm{THz}$ ). Spectroscopic parameters are taken from HITRAN ${ }^{2}$ and the JPL ${ }^{3}$ database. The pseudo-continuum water vapour absorption and collisionally-induced dry absorption are included as a quadratic frequency term according to Pardo et al. (2001) for $f<1 \mathrm{THz}$ and Borysow \& Frommhold (1986) for $f>1 \mathrm{THz}$.

This model has previously been used to calculate the atmospheric transmission up to $2000 \mathrm{GHz}(\sim 150 \mu \mathrm{m})$ for a large number of astronomical sites (Schneider et al. 2009). The results can be found on a dedicated website ${ }^{4}$. For those studies, approriate temperature and pressure profiles (for example Antarctic profiles for Dome C) of the sites were used (Schneider et al. 2009). The colder Antarctic atmosphere preferentially populates the lowlying energy state of water, leading to a greater absorption for a given PWV than that of a non-polar site. This effect is included in the model.

\subsection{Measurements, data analysis and results}

The atmospheric emission at $200 \mu \mathrm{m} S(X)$ is a function of zenith opacity $\tau_{200}$, airmass $X$ and atmospheric temperature $T_{\text {atm }}$ (see Eq. (1)). $G$ and $T_{\text {ref }}$ are the gain and the offset of the detector determined by an internal "hot-cold" calibration. $a_{\mathrm{eff}}$ is the transmission of an effective grey body at temperature $T_{\text {eff }}$ which takes in account all the noise contributions (e.g. the instrument window, see Calisse et al. 2004); these parameters were determined by an external calibration performed during the two summer campaigns. The two unknown variables $\tau_{200}$ and $T_{\text {atm }}$ can be extracted from Eq. (1) by a non linear regression of $S$ as function of the airmass.

$$
S(X)=-G\left(a_{\mathrm{eff}}\left(1-e^{-X \tau_{200}}\right) T_{\mathrm{atm}}+\left(1-a_{\mathrm{eff}}\right) T_{\mathrm{eff}}+T_{\mathrm{ref}}\right)
$$

\footnotetext{
${ }^{2}$ High Resolution Transmission molecular absorption database.

3 Jet Propulsion Laboratory.

${ }^{4}$ http://sites.google.com/site/astrosubmm
}

Table 1. Quartiles of SUMMIT08 measurements in 2008, 2009, 2010 and cumulated on the three years.

\begin{tabular}{l|c|c|c}
\hline \hline Time \% 2008 & PWV $(\mathrm{mm})$ & Trans $_{200}$ & Trans $_{350}$ \\
\hline $25 \%$ & 0.19 & 0.15 & 0.60 \\
$50 \%$ & 0.22 & 0.11 & 0.56 \\
$75 \%$ & 0.29 & 0.07 & 0.50 \\
Time \% 2009 & PWV $(\mathrm{mm})$ & Trans $_{200}$ & Trans $_{350}$ \\
\hline $25 \%$ & 0.25 & 0.09 & 0.54 \\
$50 \%$ & 0.32 & 0.05 & 0.48 \\
$75 \%$ & 0.39 & 0.03 & 0.42 \\
Time \% 2010 & PWV $(\mathrm{mm})$ & Trans $_{200}$ & Trans $_{350}$ \\
\hline $25 \%$ & 0.22 & 0.12 & 0.57 \\
$50 \%$ & 0.28 & 0.07 & 0.51 \\
$75 \%$ & 0.38 & 0.03 & 0.43 \\
Time \% cumulated & PWV $(\mathrm{mm})$ & Trans $_{200}$ & Trans $_{350}$ \\
\hline $25 \%$ & 0.21 & 0.12 & 0.58 \\
$50 \%$ & 0.27 & 0.08 & 0.52 \\
$75 \%$ & 0.35 & 0.04 & 0.45 \\
\hline
\end{tabular}

Notes. PWV and transmission at $350 \mu \mathrm{m}$ are derived using the MOLIERE model.

The $200-\mu \mathrm{m}$ filter has a bandwidth $(F W H M)$ of $9 \mu \mathrm{m}$. The measured zenith transmission is an average over the bandwidth of the filter. Using the atmospheric model MOLIERE (Sect. 2.1), we characterize the atmospheric window at a given PWV, i.e. the transmission of the atmosphere as a function of the wavelength. For example, in Fig. 1, the average value of the transmission over the bandwdith of the atmospheric window around $200 \mu \mathrm{m}$ is less than the transmission at the peak. We can convolve the transmission spectra of the filter with that of the atmospheric window and thereby evaluate how much the peak transmission is under-estimated. On average, a relative underestimation of $25 \%$ was calculated for the zenith transmission (i.e. an average transmission of $10 \%$ implies a peak transmission of $12.5 \%$ ).

MOLIERE is used to relate the opacity at any wavelength to the atmospheric PWV. For the $200-\mu \mathrm{m}$ window, for example, we extract from the transmission curves (Fig. 1) the 200- $\mu \mathrm{m}$ transmission for a given PWV. Then, we convert the transmission into an opacity and perform a linear regression of the PWV as a function of this opacity. The relation is given by Eq. (2) with a correlation coefficient of 0.9999 .

$P W V=0.13 \times \tau_{200}-0.06$.

SUMMIT08 works on a two-hour cycle, performing a measurement at airmass $X=1$ and 2 as well as two full skydips with ten pointing angles from horizon to zenith. The transmission at $350 \mu \mathrm{m}$ and the PWV were deduced using MOLIERE, with the method described above.

The points in Figs. 2-4 are average values per day, for 2008, 2009, and 2010 respectively. Quartiles of transmission at zenith for 200 and $350 \mu \mathrm{m}\left(\operatorname{Trans}_{200 / 350}=\exp \left(-\tau_{200 / 350}\right)\right)$, and PWVs are indicated in Table. 1. Quartiles represent the distribution function of PWV content, e.g. there is less than $0.19 \mathrm{~mm}$ of PWV at Dome C, $25 \%$ of the time that SUMMIT08 was working in 2008.

The quartiles indicate that there was a degradation of the transmission between 2008 and 2009 as the amount of PWV increased significantly in one year.

Opacity variations based on a one-day avearges are plotted in Fig. 5. The standard deviation of the opacity distribution is 0.45 , which corresponds to variations of only $37 \%$ in transmission. Atmospheric conditions are very stable during winter in 


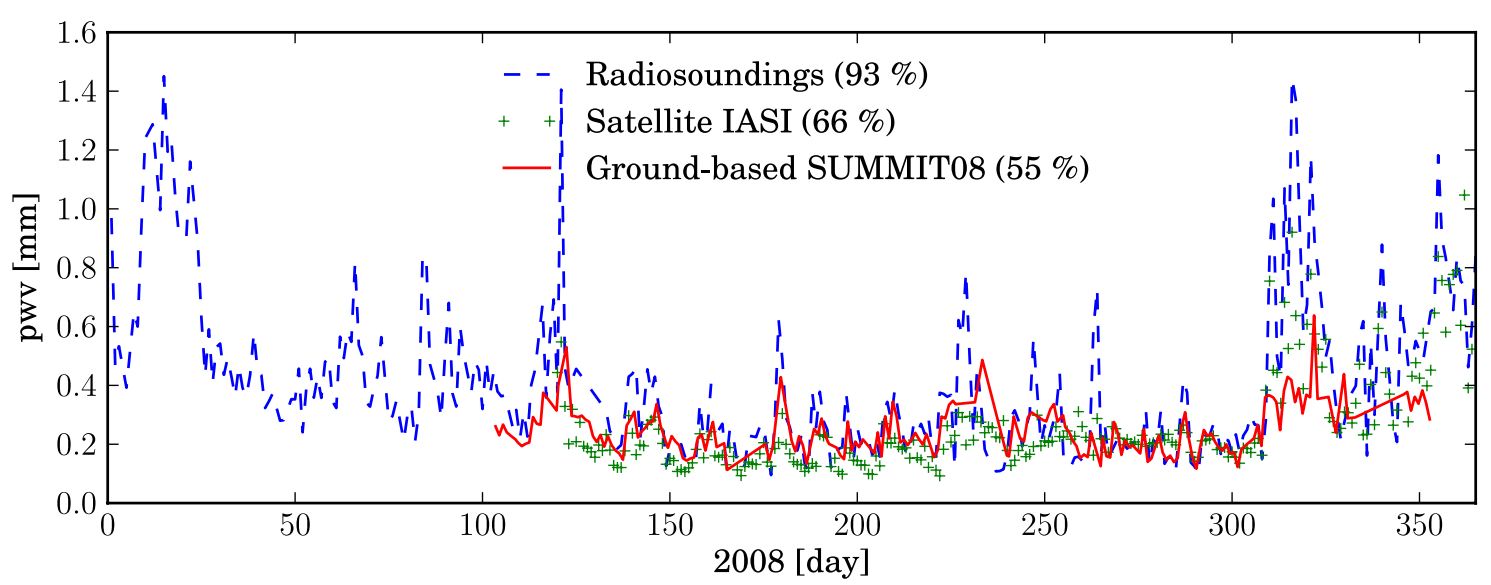

Fig. 2. Precipitable Water Vapour (PWV) in [mm] in 2008 at the Concordia Station measured in situ by SUMMIT08 (red line), by radiosoundings (blue line) and from space by IASI (green line). Percentages indicate the fraction of time that each of the instruments worked during the year.

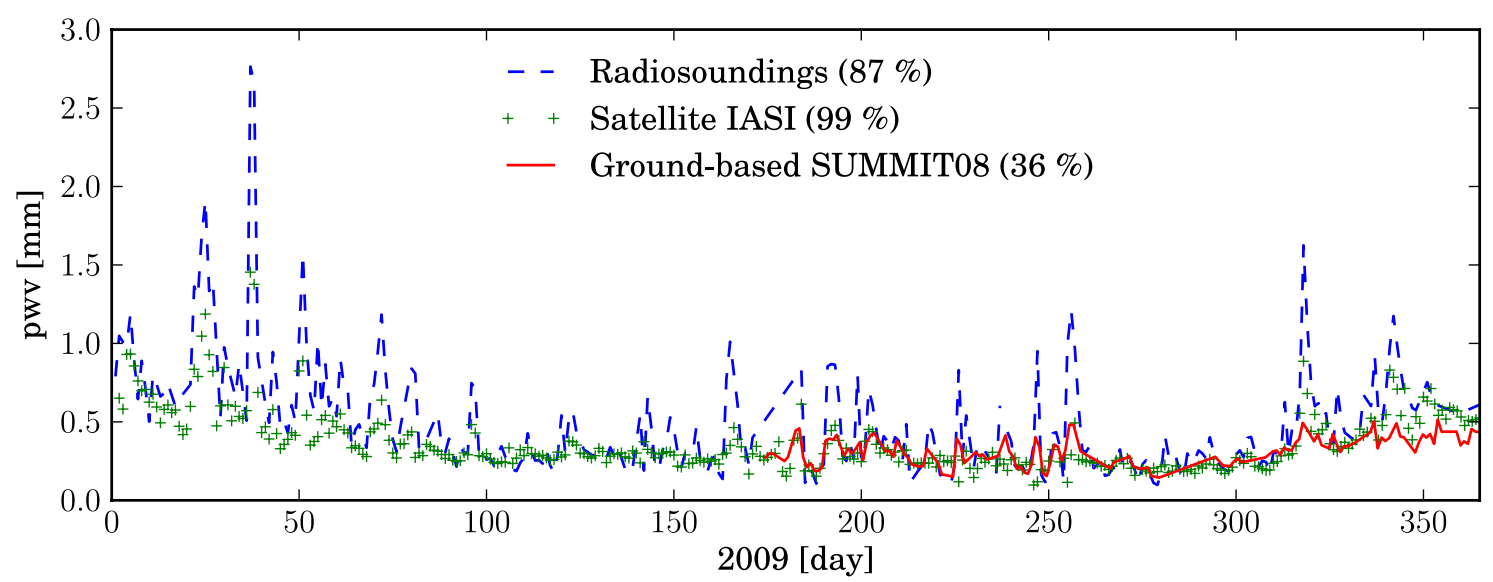

Fig. 3. PWV in 2009 at the Concordia Station measured in situ by SUMMIT08 (red line), by radiosoundings (blue line) and from space by IASI (green line).

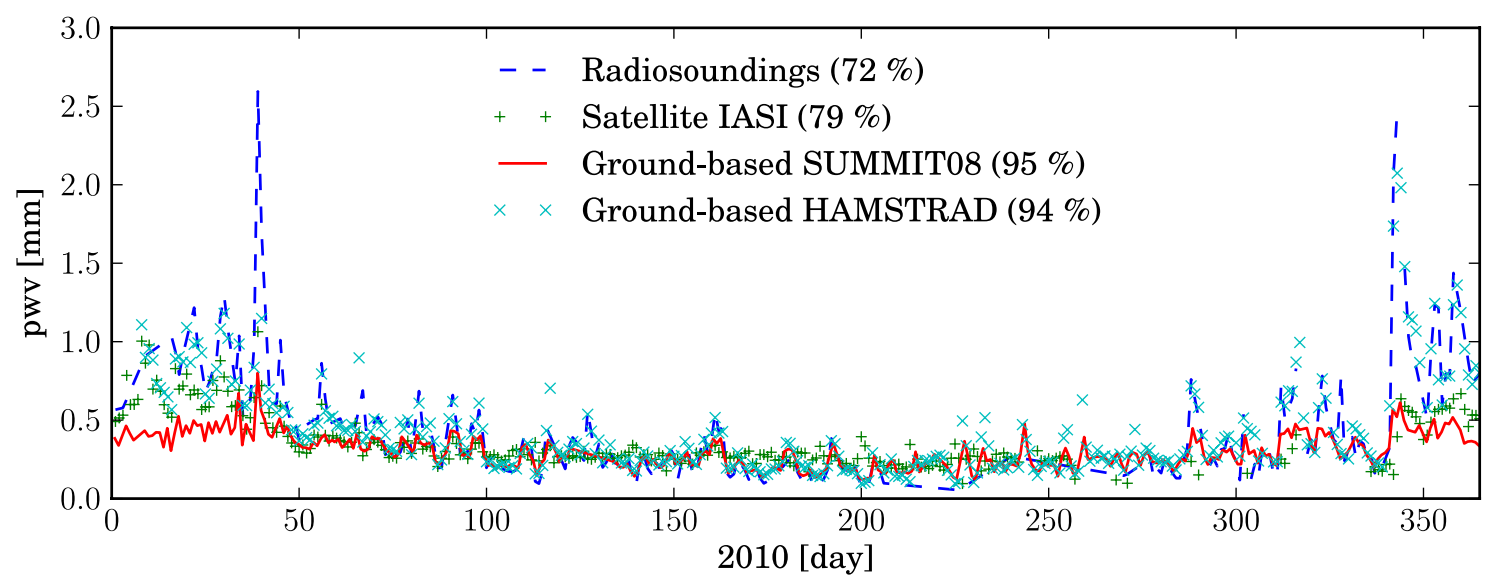

Fig. 4. PWV in 2010 at the Concordia Station measured in situ by SUMMIT08 (red line), by radiosoundings (blue line), by HAMSTRAD (turquoise line) and from space by IASI(green line).

Antarctica, where the polar night avoids the strong diurnal variations of water vapour induced by the solar radiation in non-polar regions.

\section{Comparisons between instruments}

\subsection{Satellite measurements : IASI}

IASI (Infrared Atmospheric Sounding Interferometer) is an atmospheric interferometer working in the infrared, launched in
2006 on the METOP-A satellite (Phulpin et al. 2007; Pougatchev et al. 2008). The data are available at the website of the Centre for Atmospheric Chemistry Products and Services ${ }^{5}$. IASI was developed by the French Space Agency $\mathrm{CNES}^{6}$ in collaboration with EUMETSAT $^{7}$. The satellite is in a polar orbit such that each

\footnotetext{
5 http://ether.ipsl.jussieu.fr

6 Centre National d'Études Spatiales.

7 European organisation for meteorological satellites http: //www . eumetsat. int
} 


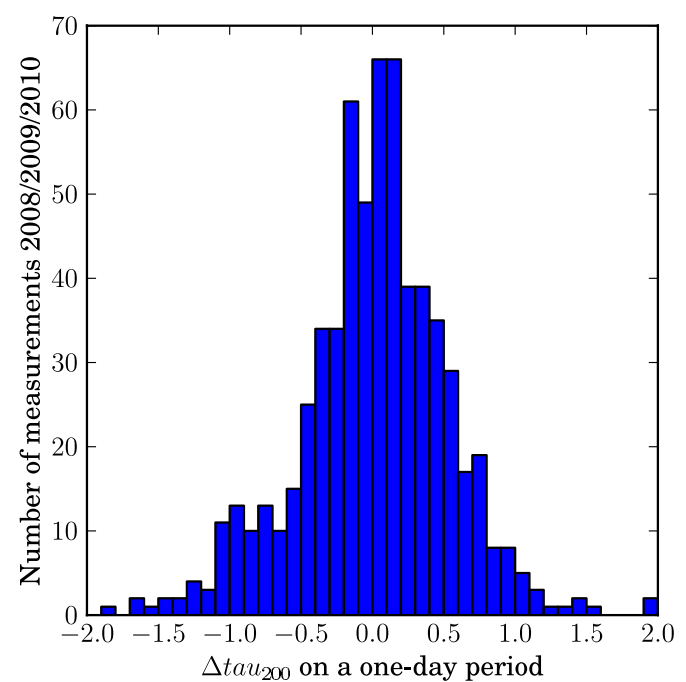

Fig. 5. Distribution of $200-\mu \mathrm{m}$ opacity variations on a one-day statistic for $2008 / 2009 / 2010$.

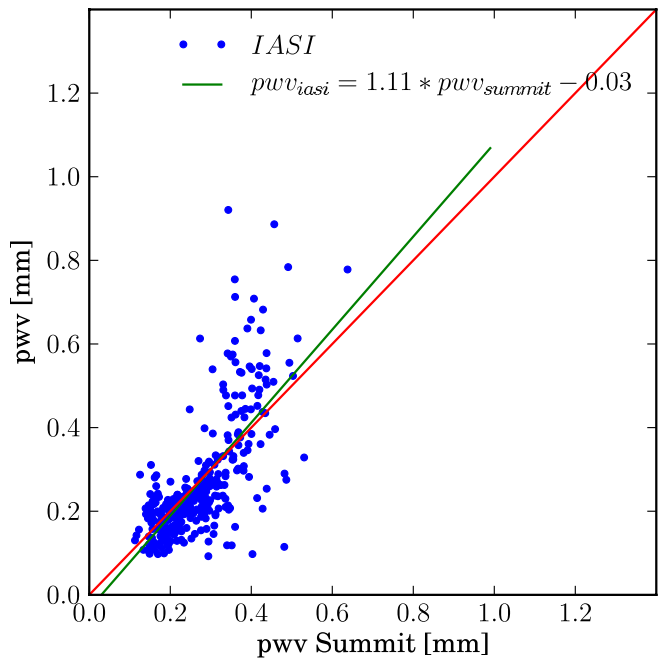

Fig. 6. Correlation between in situ measurement (SUMMIT08) and satellite measurments(IASI) between 2008 and 2010.

point on Earth is seen at least once a day by the detector. IASI is a Fourier transform spectrometer (FTS) working between 3.7 and $15.5 \mu \mathrm{m}$. It is associated with an infrared imager, operating between 10.3 and $12.5 \mu \mathrm{m}$. Each pixel of the instrument corresponds to a spatial extent of $12 \mathrm{~km}$ at nadir, and vertical profiles of humidity at ninety altitude levels are retrieved with a typically $10 \%$ accuracy. The amount of precipitable water vapour is given by the integral of these vertical profiles. We took all the measurements in a zone of $110 \mathrm{~km}^{2}$ around Concordia. The results for 2008, 2009, and 2010 are plotted together with the SUMMIT08 data in Figs. 2-4 together with the correlation of the data in Fig. 6. There is a small negative bias for SUMMIT08 (SUMMIT08 values are lower than the ones for IASI) at low PWV (less than $0.4 \mathrm{~mm}$ ), and a significant negative bias at high PWV (more than $0.4 \mathrm{~mm}$ ). This bias occurs during the summer period, when the PWV is high. An analysis of the skydips shows that the fluxes received at different elevations are highly variable during the summer and do not fit the exponential profile given by Eq. (1). Therefore we conclude that there are atmospheric temperature or PWV variations at the scale of Summit field of view that pollute the analysis and prevent us to extract an accurate value for the PWV during summer periods.

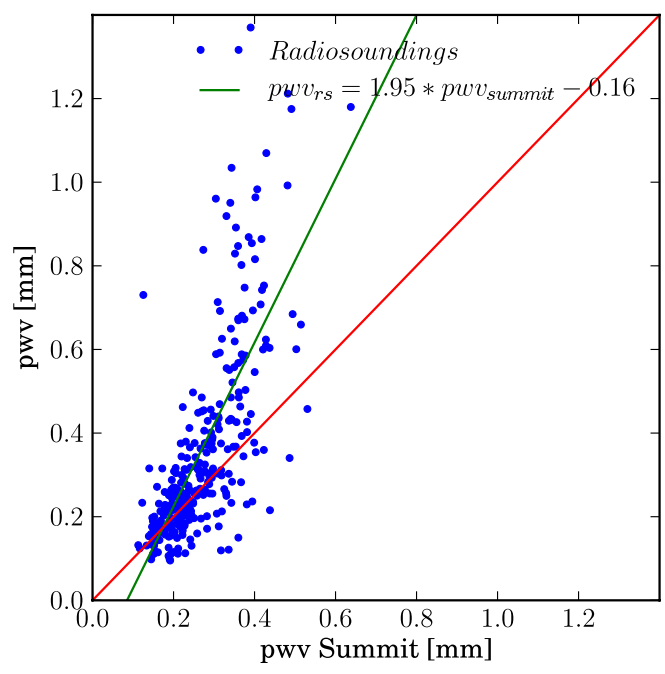

Fig. 7. Correlation between in situ measurements SUMMIT08 and radiosoundings between 2008 and 2010.

\subsection{In situ measurements: radiosoundings and HAMSTRAD}

An atmospheric radiosounding station was installed at Concordia Station in 2005. Radiosonde balloons are launched twice a day, and reach an altitude of $25-30 \mathrm{~km}$. The vertical profile of the atmospheric pressure, temperature and relative humidity is measured during the ascent. PWV is calculated by integrating this vertical profile. The data and additional information were obtained from IPEV/PNRA Project "Routine Meteorological Observation at Station Concordia" (see Tomasi et al. 2006). The results from these measurements are plotted in Fig. 2-4 and the correlation of the data with SUMMIT08 data in Fig. 7.

HAMSTRAD is a microwave instrument operating at 60 and $183 \mathrm{GHz}$, measuring temperature and water vapour profiles between 0 and $10 \mathrm{~km}$ with a time resolution of $7 \mathrm{~min}$ (Ricaud et al. 2010). The instrument has been fully operating since the beginning of 2010. PWV derived from the vertical profile is plotted in Fig. 4, and the correlation of these data with the SUMMIT08 data in Fig. 8. The SUMMIT08 measurements and radiosonde or HAMSTRAD data are well correlated at low PWV (less than $0.4 \mathrm{~mm}$ ), but less so at high PWV (more than $0.4 \mathrm{~mm}$ ), probably due to temperature or PWV spatial variations during summer skydips of SUMMIT08.

\subsection{Instrument and site comparison}

Low PWV periods mainly occur during the austral winter (from March to October) and high PWV periods are concentrated during austral summer periods (from November to February). In contrast to, for example, the Chilean sites where strong day-today variations are found, there is a very long and stable period of high transparency at submillimetre wavelengths at Dome C. This will be the subject an upcoming paper, in which we study a large number of locations using satellite data to characterize the quality of the sites in an unbiased way. The stable periods at Dome C are identified as winter periods in the quartiles (Fig. 9). Summer period quartiles are plotted in Fig. 10, and total quartiles per period in Fig. 11. The time fractions used for these quartiles are based on the time the instruments were in operation. The

8 http://www.climantartide.it 


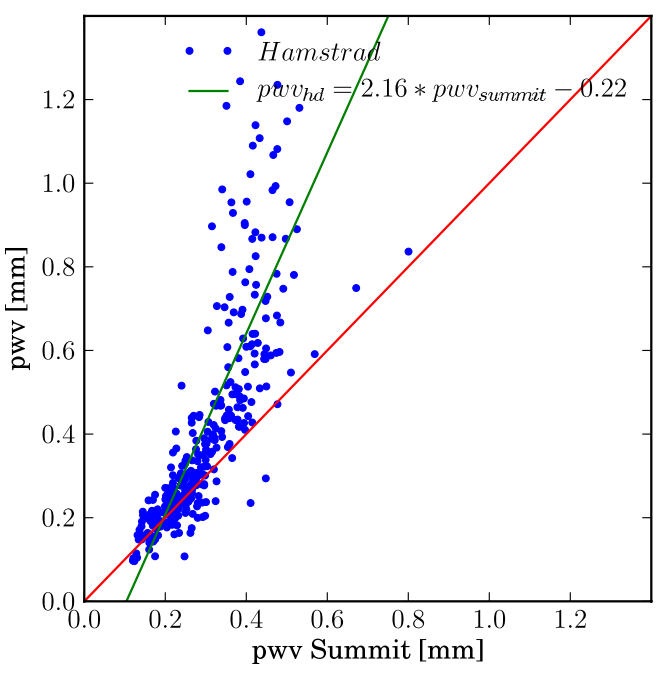

Fig. 8. Correlation between in situ measurements SUMMIT08 and HAMSTRAD in 2010.
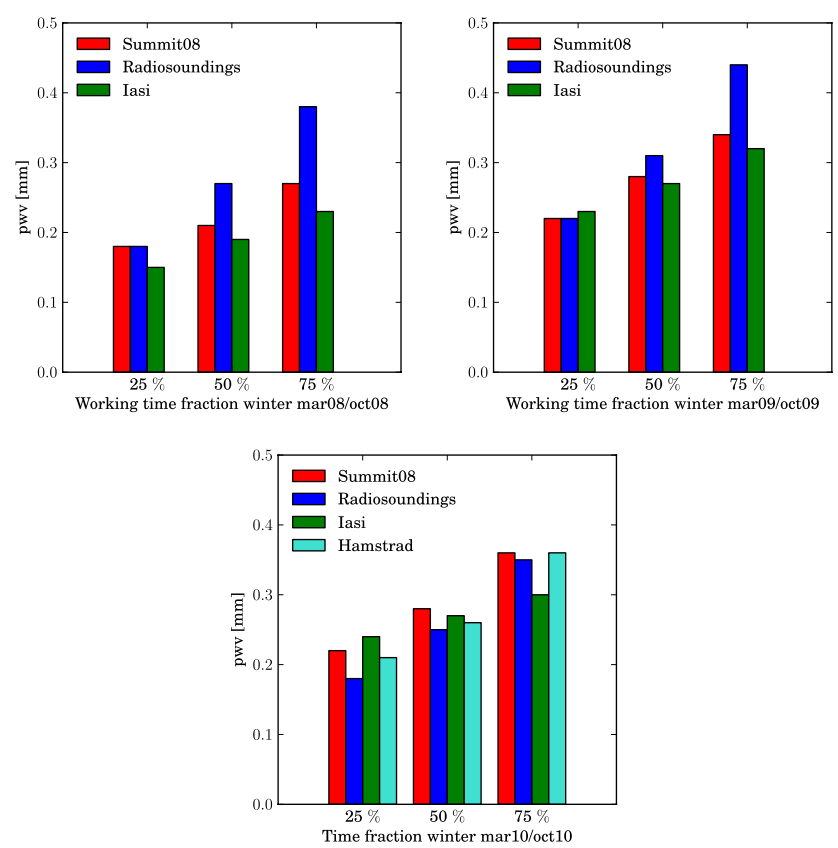

Fig. 9. Winter quartile during the working time of the instruments between March 2008 and October 2010.

quartiles of SUMMIT08 are slightly biased because it was not functional all the time during these periods (between $36 \%$ and $95 \%$ ), while the satellite and radiosounding quartiles are less biased (IASI was operational between $85 \%$ and $100 \%$ of the time during these periods).

The SUMMIT08 campaign allows validation of the satellite measurements. There is a very good correlation during winter periods between IASI, SUMMIT08, HAMSTRAD, and radiosonde data. However the correlation is not so good during summer periods, or more generally when PWV is high (more than $0.4 \mathrm{~mm}$ ). HAMSTRAD is well correlated with the radiosonde data but there is a dry bias for IASI data. This bias was previously identified by Ricaud et al. (2010) and could be attributed to the influence of the surface emission parameter over Antarctica. In order to further improve the effectiveness of satellites for measuring temperature and humidity over the White
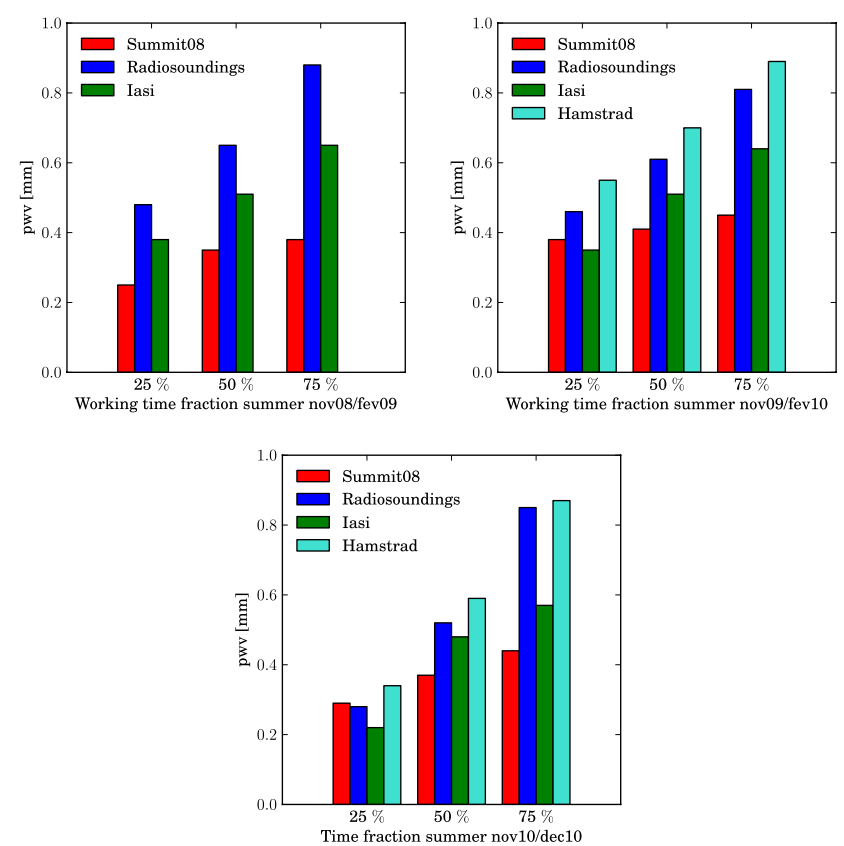

Fig. 10. Summer quartile during the working time of the instruments between March 2008 and December 2010.
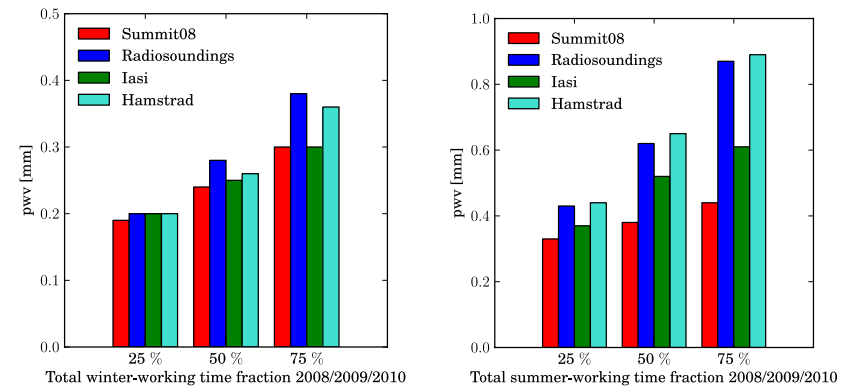

Fig. 11. Total quartile per summer/winter periods during the working time of the instruments between March 2008 and December 2010.

Continent, a consortium (see Rabier et al. 2010) was created to collect ground-based data for comparison; in particular to understand why measurements differ at high PWV.

Nevertheless, for astronomical observations, we are only interested in PWV values below about $0.3 \mathrm{~mm}$ (50\% transmission at $350 \mu \mathrm{m}$ wavelength), and the disparity between measurements at high PWV disappears for the median and $25 \%$ winter time fraction (Figs. 9 and 11).

\section{Polar constraints}

\subsection{Frost formation}

The GIVRE experiment was designed in 2006 to study frost formation (Durand et al. 2008). It consists of a series of twelve probes of different shapes and colors: five black and two bright cylinders, two black and two bright disks, and an aluminium plate. The probes are divided in two groups, which were placed at different altitudes on a fifty meter tower. They can all be warmed by heaters except for the plate, which instead can have dry air (from near the ground) blown over it. In 2007, building upon the results from the first year of tests, an antifrost system was installed on the COCHISE telescope (Sabbatini et al. 2009) 


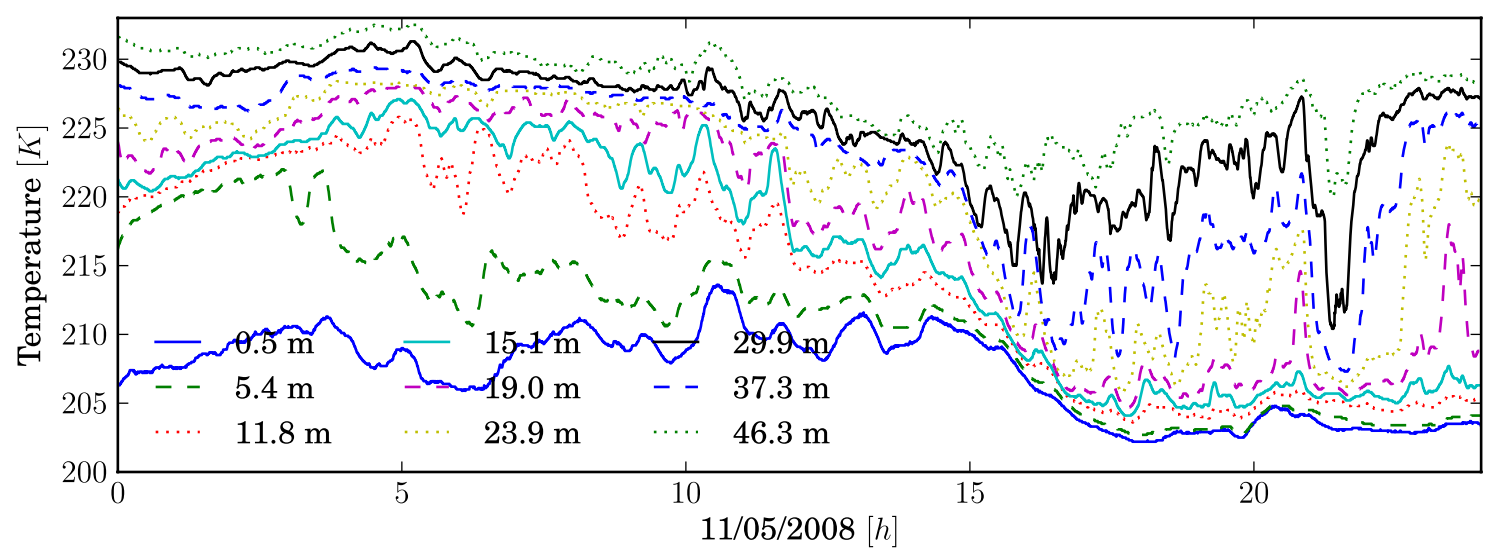

Fig. 12. Typical temperature variation at altitudes between $0.5 \mathrm{~m}$ and $46.3 \mathrm{~m}$ during a winter day. Each curve is the temperature in Kelvin measured at a given altitude on the $50 \mathrm{~m}$ tower

Table 2. Water vapour partial pressure for saturated air as a function of atmospheric temperature.

\begin{tabular}{cc}
\hline \hline Temperature $\left({ }^{\circ} \mathrm{C}\right)$ & Partial pressure of water vapour $(\mathrm{Pa})$ \\
\hline-40 & 12.84 \\
-50 & 3.94 \\
-60 & 1.08 \\
-70 & 0.26 \\
\hline
\end{tabular}

at Concordia. Three different techniques are used : heaters at the rear of the mirror, an infrared lamp, and an air blowing system.

During the summer season, the mean temperature at Concordia is of the order of $-30{ }^{\circ} \mathrm{C}$ and no frost appears on instruments during this period. However during winter, the temperature can fall to $-70^{\circ} \mathrm{C}$ and the partial pressure of water vapour is two orders of magnitude lower (see Table 2). At $-70^{\circ} \mathrm{C}$, a partial pressure of water vapour of $0.26 \mathrm{~Pa}$ corresponds to an absolute humidity of $1 \mathrm{mg}$ of water per $\mathrm{m}^{3}$; i.e., a hundred times smaller than a drop of water. In such conditions, however, the relative humidity is very high, nearly $100 \%$. If the temperature decreases by $5{ }^{\circ} \mathrm{C}$, the relative humidity will increase by almost a factor of two and can become much higher than $100 \%$, leading to ice nucleation and frost formation. Frost can also appear due to snow precipitation on surfaces.

Three systems can be used against frost formation and snow deposit:

- Warming up the probes by three or four degrees is enough to prevent frost. It is also the most efficient way to prevent frost formation on the mirror of a telescope.

- Taking dry air at snow surface level and blowing it over the probes is effective; it is also a useful method because it does not require warm optics (which can introduce undesirable temperature gradients).

- Explosive air blowing can be used to physically remove the snow deposit.

Frost mainly forms as a result of the cooling of a surface radiating towards the sky. Close to these surfaces, the maximum amount of water vapour that the atmosphere can hold is lower and frost appears. Warming up these surfaces by three or four degrees is sufficient to compensate for the heat loss due to radiation. Blowing dry air taken from close to the ground is an also effective way to dehumidify the local environment.

\subsection{Temperature gradient in the boundary layer}

The large temperature gradient in the first thirty meters above the snow limits the choice of materials that can be used to build large instruments in a polar environment. The MAST experiment, installed in 2007 at Concordia, consists of sixteen temperature probes placed on a fifty-meter tower (Durand et al. 2008) with a logarithmically increasing spacing bewteen the probes The probes are Pt1000 sensors sandwiched between two bright plates to protect them from solar radiation. GIVRE and MAST can be controlled from the station, and data are automatically sent daily to the station and then to Europe via the station's communication system.

Close to the ground, the atmosphere is cooled by contact with the snow surface. The temperature increases with altitude up to a point of inversion, above which the atmosphere has the usual tropospheric profile, decreasing by $6.5^{\circ} \mathrm{C}$ per kilometre. The atmosphere between the ground and the point of inversion is called the boundary layer. The thickness of this layer can be very low, leading to very good astronomical seeing in the visible (see Fossat 2005). However this thickness is variable and is responsible for strong (vertical and temporal) temperature gradients close to the ground. These gradients can be extreme, as shown in Figs. 12 and 13. The typical variations during a winter day can result in a vertical gradient of $30{ }^{\circ} \mathrm{C}$ over $50 \mathrm{~m}$, and variations of $15^{\circ} \mathrm{C}$ in one hour have been recorded. However, the engineering issues this creates can be readily overcome by using materials with low thermal expansion.

\section{Discussion and conclusions}

The necessary requirements for the future deployment of a large (10-25 m) telescope have been explored between 2007 and 2010. The atmospheric opacity at $200 \mu \mathrm{m}$ was monitored for three years, making this the first extended study at this wavelength and providing the first multi-year statistics. Based on these atmospheric opacity measurements and the derived PWV, we can conclude that the submm/ THz atmospheric windows open during a relatively large and stable fraction of time, and particularly for all windows down to $350 \mu \mathrm{m}$. The $200-\mu \mathrm{m}$ window opens for somewhat less than $25 \%$ of the time (PWV < $0.2 \mathrm{~mm}$ ). These findings are based upon a $\sim 3$ year study in which we noticed significant variations from one year to another.

Comparisons between Dome C and other sites (e.g. Dome A, Chajnantor) should be made over the same period of time to 


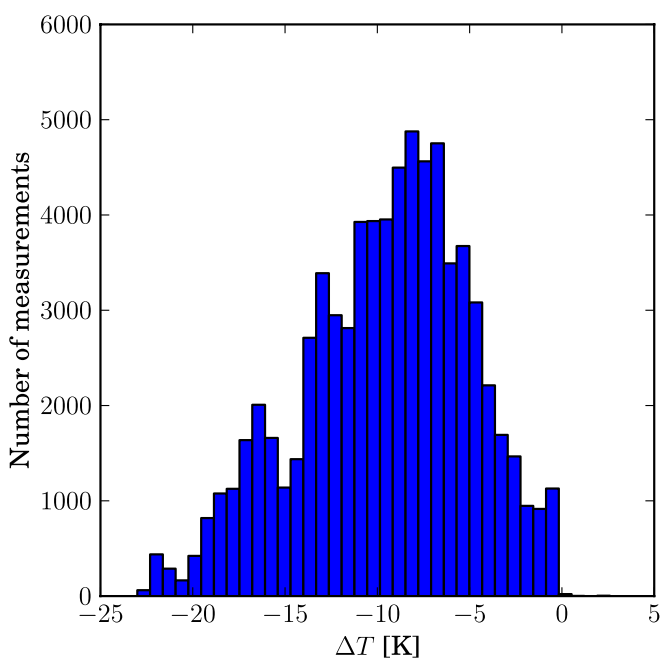

Fig. 13. Distribution of temperature gradient between $0.5 \mathrm{~m}$ and $46.3 \mathrm{~m}$ between 01/2010 and 04/2010.

be directly comparable. In addition, the issue of calibration (window transmission, filter bandwdith, etc. see Radford 2009) usually complicates comparisons between sites where measurements are made with different instruments. However, although non-polar sites can offer very good conditions for submillimetre observations (e.g. Chajnantor Radford et al. 2008), Antarctica provides not only better transmission, but also extremely good stability that is invaluable for time-series studies at submillimetre wavelengths.

In addition to the measurement of the atmospheric opacity, a knowledge of the polar constraints is an equally necessary prerequisite for operating in the harsh Antarctic plateau environment. The thermal gradient in the boundary layer and the problem of ice formation have been extensively monitored. Techniques for preventing or removing ice have also been implemented on site. Solutions for preventing and removing icing and frost proved to be useful on probes and on the surface of a $2.5-\mathrm{m}$ diameter telescope, which could scale-up for installations on larger and more complex instruments. Another polar constraint of importance is the thermal gradient that was measured in a 46-m high layer above the ground. Radford (2009) pointed out the possible lack of surface accuracy of mirrors due to thermal gradients arising from both the vertical gradient and iced surface of the telescope. Tests of image quality while removing frost will be done on the IRAIT telescope (Tosti 2006) that is currently deployed at Dome C. Further studies of the polar constraints based on the thermal gradient and variation will be discussed in technical reports. For the scope of this paper, we may conclude that any instrument in the boundary layer should be built with materials whose thermal expansion coefficient is low at temperatures around $-70{ }^{\circ} \mathrm{C}$. In addition, materials must be able to be cooled rapidly to very low temperature without internal stresses being created.

In conclusion, Dome $\mathrm{C}$ is currently one of the best accessible sites on Earth in terms of transmission in the FIR/submm. The $200-\mu$ m window opens at a level of $10-15 \%$ for slightly less than $25 \%$ of the time. Further analysis indicates that observations at 350 and $450 \mu \mathrm{m}$ would be possible all year round. These very good astronomical conditions in terms of atmospheric transmission and stability offer a unique opportunity for high angular resolution and time series observations at Dome $\mathrm{C}$ with medium size instruments.

Acknowledgements. We acknowledge the strong support of the French and Italian polar institutes IPEV and PNRA that make this research project possible. We also acknowledge the European network ARENA. The original SUMMIT instrument was designed and built by Jeff Peterson and Simon Radford.

\section{References}

André, P., Men'shchikov, A., Bontemps, et al. 2010, A\&A, 518, L102

Aristidi, E., Agabi, K., Azouit, M., et al. 2005, A\&A, 430, 739

Borysow, A., \& Frommhold, L. 1986, ApJ, 311, 1043

Calisse, P. G., Ashley, M. C. B., Burton, M. G., et al. 2004, PASA, 21, 256

Carlstrom, J. E., Ade, P. A. R., Aird, K. A., et al. 2011, PASP, 123, 568

Chamberlin, R. 2004, PASA, 21, 264

Durand, G. A., Minier, V., Lagage, P. O., et al. 2008, SPIE, 7012, 70122B

Fossat, E. 2005, JApA, 26, 349

Hills, R. E., Kurz, R. J., \& Peck, A. B. 2010, SPIE, 7733, 773317

Lawrence, J. S., Ashley, M. C. B., Tokovinin, A., \& Travouillon, T. 2004, Nature, 431, 278

Marrone, D., Blundell, R., Tong, E., et al. 2005, STT, Göteborg, Sweden, 64

Matsushita, S., Matsuo, H., Pardo, J., \& Radford, S. 1999, PASJ, 51, 603

Minier, V., Olmi, L., Lagage, P. O., et al. 2008, EAS, 33, 21

Minier, V., Olmi, L., Durand, G. A., et al. 2009, EAS, 40, 269

Oberst, T. E., Parshley, S. C., Stacey, G. J., et al. 2006, ApJ, 652, L125

Pardo, J., Cernicharo, J., \& Serabyn, E. 2001, ITAP, 49, 1683

Peterson, J., Radford, S., Ade, P., et al. 2003, PASP, 115, 383

Phulpin, T., Blumstein, D., Prel, F., et al. 2007, SPIE, 6684, 66840

Pilbratt, G. L., Riedinger, J. R., Passvogel, T., et al. 2010, A\&A, 518, L1

Pougatchev, N., August, T., Calbet, X., et al. 2008, SPIE, 7081, 70810

Rabier, F., Bouchard, A., Brun, E., et al. 2010, BAMS, 91, 69

Radford, S. J. E. 2009, EAS, 40, 405

Radford, S. J. E., Giovanelli, R., Gull, G. E., \& Henderson, C. P. 2008, SPIE, 7012, 70121

Ricaud, P., Gabard, B., Derrien, S., et al. 2010, ITGRS, 48, 1365

Sabbatini, L., Dall'Oglio, G., Pizzo, L., Miriametro, A., \& Cavaliere, F. 2009, EAS, 40,319

Saunders, W., Lawrence, J. S., Storey, J. W. V., et al. 2009, PASP, 121, 976

Schneider, N., Urban, J., \& Baron, P. 2009, P\&SS, 57, 1419

Schneider, N., Motte, F., Bontemps, S., et al. 2010, A\&A, 518, L83

Stark, A. A., Bally, J., Balm, S. P., et al. 2001, PASP, 113, 567

Tomasi, C., Petkov, B., Benedetti, E., et al. 2006, JGR, 111, D20305

Tosti, G. 2006, SPIE, 6267, 62671

Urban, J., Baron, P., Lauti, N., et al. 2004, JQSRT, 83, 529

Valenziano, L., \& Dall’Oglio, G. 1999, PASA, 16, 167

Yang, H., Kulesa, C. A., Walker, C. K., et al. 2010, PASP, 122, 490 